Supporting Information for

\title{
Interplay of Charged States and Oxygen Dissociation Induced by Vacancies in Phosphorene
}

\author{
Fangyang Zhan ${ }^{1}$, Wangping Xu ${ }^{1,2}$, Runling Zou ${ }^{1}$, Jiali Yang ${ }^{1}$, Jing Fan ${ }^{3}$, Xiaozhi Wu ${ }^{1}$, \\ and Rui Wang ${ }^{1, *}$ \\ ${ }^{1}$ Institute for Structure and Function \& Department of Physics, Chongqing University, Chongqing \\ 400044, People's Republic of China. \\ ${ }^{2}$ Department of Physics and Institute for Quantum Science and Engineering, Southern University \\ of Science and Technology, Shenzhen 518055, People's Republic of China. \\ ${ }^{3}$ Center for Computational Science and Engineering, Southern University of Science and \\ Technology, Shenzhen 518055, People's Republic of China.
}

In this Supporting Information, several possible structures of $\mathrm{O}_{2}$ dissociation in pristine and defective phosphorene and the reaction of one $\mathrm{O}_{2}$ molecule for the double vacancy are included.

\section{Corresponding Author:}

*R.W.: rcwang@,cqu.edu.cn 
(a)

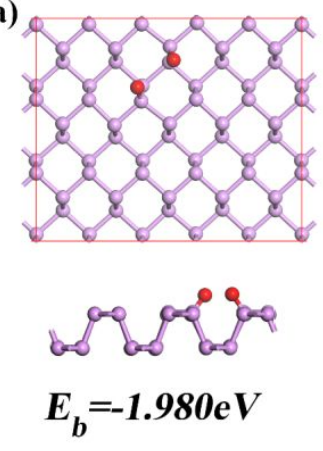

(b)
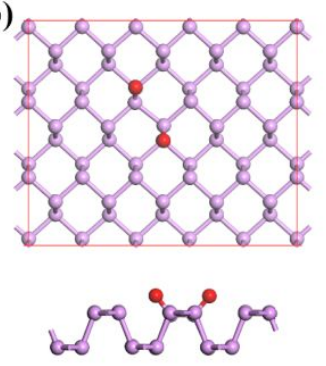

$E_{b}=-2.057 \mathrm{eV}$ (c)
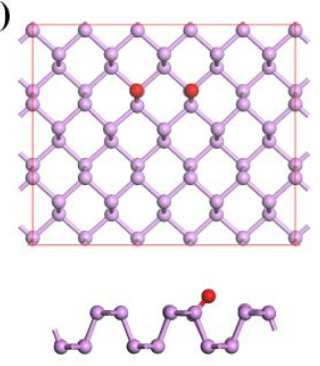

$E_{b}=-2.066 \mathrm{eV}$ (d)
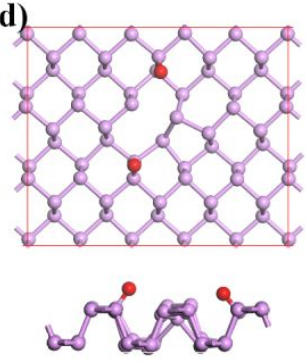

$E_{b}=-2.074 \mathrm{eV}$

(h)
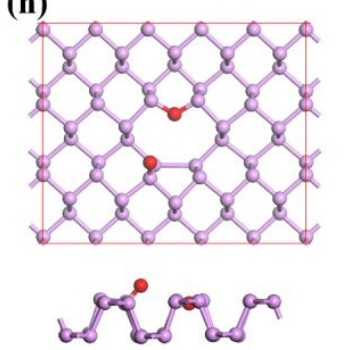

$$
E_{b}=-2.603 \mathrm{eV}
$$

(I)
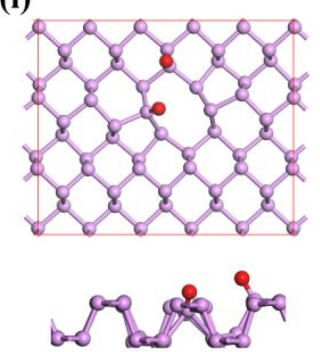

$$
E_{b}=-2.077 \mathrm{eV}
$$

(e)
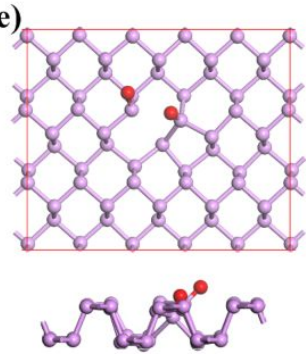

$$
E_{b}=-2.094 \mathrm{eV}
$$

(i)
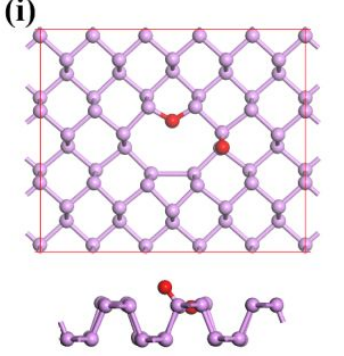

$$
E_{b}=-2.612 \mathrm{eV}
$$

(m)
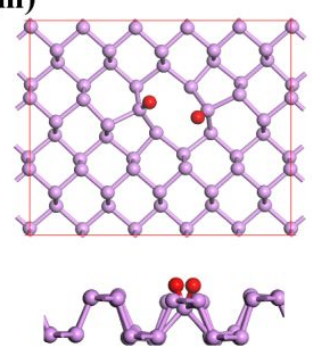

(f)
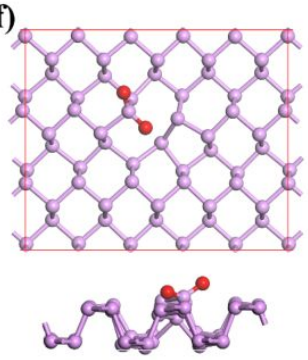

$$
E_{b}=-2.315 \mathrm{eV}
$$

(j)

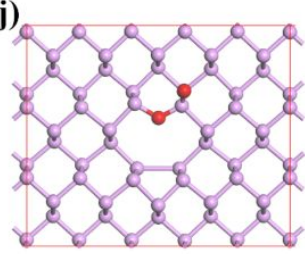

$$
\begin{aligned}
& E_{b}=-2.905 \mathrm{eV}
\end{aligned}
$$

(n)

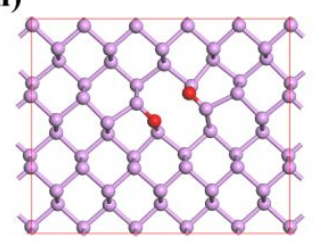

(k)
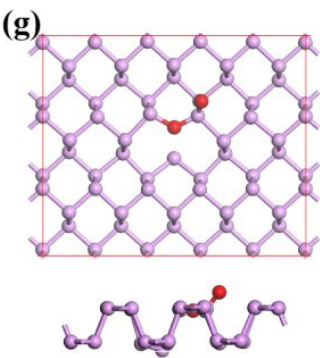

$$
E_{b}=-2.475 \mathrm{eV}
$$
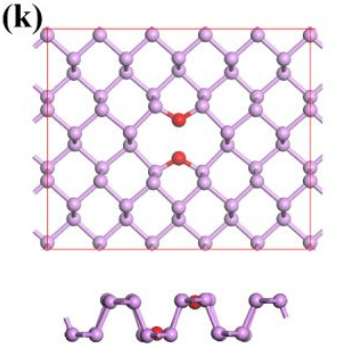

$E_{b}=-3.118 \mathrm{e} V$

(o)
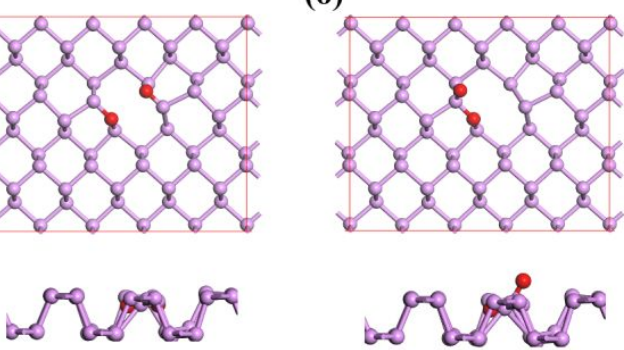

$$
E_{b}=-2.477 \mathrm{eV}
$$

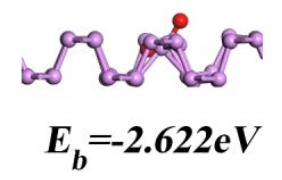

Figure S1: Top and side views for several possible structures of $\mathrm{O}_{2}$ dissociation in (a)-(c) pristine, (d)-(g) SV(5|9), (h)-(k) DV(5|8|5)-I, and (l)-(o) DV(5|8|5)-II defective phosphorene. The corresponding binding energies for an oxygen atom are also shown. 


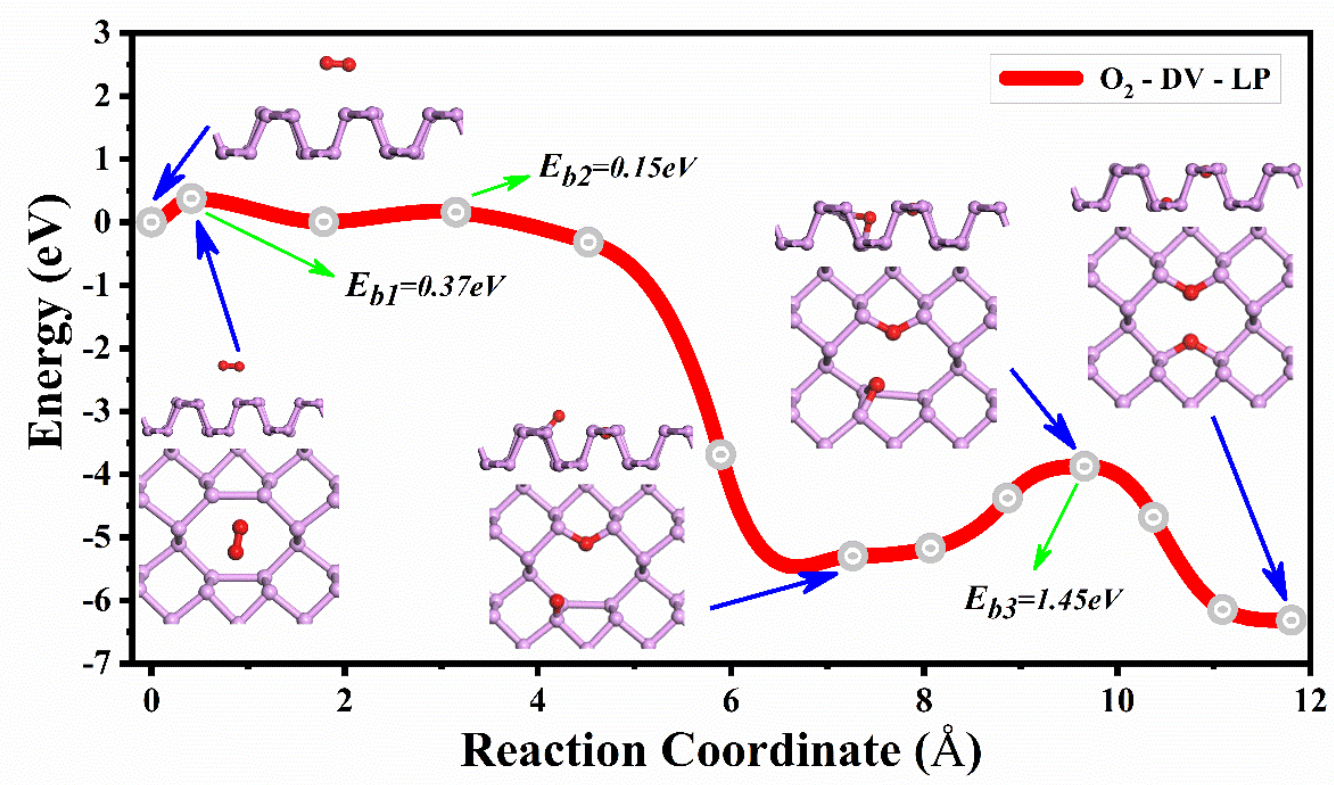

Figure S2: The reaction path of one $\mathrm{O}_{2}$ molecule for DV(5|8|5)-I.

As shown in Fig. S2, the $\mathrm{O} 2$ molecule dissociating into two individual $\mathrm{O}$ atoms needs to overcome an energy barrier of $0.37 \mathrm{eV}$. Subsequently, one $\mathrm{O}$ atom penetrates into the upper vacancy, bonding adjacent $\mathrm{P}$ atom and forming an oxygen bridge. The other $\mathrm{O}$ atom forms a dangling O-P bond. Eventually, the dangling O-P bond crosses through the upper hollow channel and then bonds the lower $\mathrm{P}$ atom forming an oxygen bridge. This process will overcome a high energy barrier $1.45 \mathrm{eV}$. Obviously, this mechanism is not compatible with rapid degradation of phosphorene in ambient conditions. 